\title{
On the Asymptotic Idealness of the Asmuth-Bloom Threshold Secret Sharing Scheme
}

\author{
Constantin Cătălin Drăgan * and Ferucio Laurenţiu Ţ̧iplea \\ Department of Computer Science \\ "Al.I.Cuza" University of Iaşi \\ Iaşi 700506, Romania \\ e-mail: \{fltiplea,constantin.dragan\}@info.uaic.ro
}

\begin{abstract}
A necessary and sufficient condition for the asymptotic idealness of the AsmuthBloom threshold secret sharing scheme is proposed. Apart from this, a comprehensive analysis of the known variants of the Asmuth-Bloom threshold secret sharing scheme is provided, clarifying the security properties achieved by each of them.
\end{abstract}

\section{Introduction and Preliminaries}

A $(t+1, n)$-threshold secret sharing scheme $((t+1, n)$-threshold scheme, for short $)$ is a method of partitioning a secret among $n$ users by providing each user with a share of the secret such that any $t+1$ users can uniquely reconstruct the secret by pulling together their shares. If less than $t+1$ shares give no information about the secret, from an information theoretic point of view, then the scheme is called perfect. In addition to this, if the share spaces have the same dimension with the secret space, the scheme is called ideal.

One of the techniques to construct threshold schemes is based on the Chinese Remainder Theorem (CRT) [1-5]. The main idea is to use sequences of pair-wise coprime positive integers with special properties. The shares are obtained by dividing the secret or a secret-dependent quantity by the numbers in the sequence and collecting the remainders.

The CRT-based threshold schemes proposed so far are neither perfect nor ideal. However, they offer some security degree and, in order to study it, Quisquater et al. [6] have introduced the concepts of asymptotic perfectness and asymptotic idealness. They also proved that the threshold scheme in [3] is asymptotically ideal (and, therefore, asymptotically perfect) provided that it uses sequences of consecutive primes and the secret is uniformly chosen from the secret space. This result was later improved in [4] by showing that the asymptotic idealness of this scheme is achieved for a subclass of compact sequences of co-primes [4]. Compact sequences of co-primes capture very well the idea of sequence of numbers of the "same magnitude", and they are much denser than sequences of consecutive primes [4]. Moreover, [4] studies the security of the

\footnotetext{
* Constantin Cătălin Drăgan was supported by the European Social Fund in Romania, under the responsibility of the Managing Authority for the Sectoral Operational Programme for Human Resources Development 2007-2013 [grant POSDRU/CPP 107/DMI 1.5/S/78342]
} 
Asmuth-Bloom threshold scheme [1] and also proposes some asymptotically perfect and ideal variants of it. Another variant of the Asmuth-Bloom threshold scheme was proposed in [7] which provides better security than the original Asmuth-Bloom threshold scheme.

Contribution This paper comes to complete a series of results regarding the security of CRT-based threshold schemes $[6,4,5]$. The main result of the paper is a necessary and sufficient characterization of the security of the Asmuth-Bloom threshold scheme (Theorem 1). Thus, we show that this scheme is asymptotically ideal with respect to the uniform distribution on the secret space if and only of it is based on 1-compact sequences of co-primes. We believe that this result is important from two points of view: first, it closes completely the security problem of the Asmuth-Bloom threshold scheme, and secondly it emphasizes the importance of 1-compact sequences of co-primes in studying the security of the CRT-based secret sharing schemes. Apart from the above main result, our paper makes a comprehensive analysis of the Asmuth-Bloom threshold scheme variants proposed so far, clarifying and discussing the security properties achieved by each of them.

A similar characterization result to our Theorem 1 was obtained in [5] for the Goldreich-Ron-Sudan threshold scheme [3]. However, the proof of our Theorem 1 cannot be obtained as in [5] mainly because the estimate of the loss of entropy in [5] is to weak for the case of the Asmuth-Bloom threshold scheme (see also Section 4 in [4]). That is why in this paper we provide first a very precise estimate of the loss of entropy for the Asmuth-Bloom threshold scheme (Lemma 2), from which the upper bound in $[6,5]$ immediately follows.

Paper structure The paper is structured into six sections. The first one is an introduction to the theory of CRT-based threshold schemes. It also establishes the basic notation and terminology. The second section discusses the variants of the AsmuthBloom threshold scheme met in the literature. The basic security properties a CRTbased threshold scheme should fulfill are recalled in the third section. Moreover, an important result regarding the loss of entropy in a threshold scheme is also obtained. The fourth section establishes our main result regarding the security of the AsmuthBloom threshold scheme, while the fifth section clarifies the security of some variant of the Asmuth-Bloom threshold scheme. We conclude in Section 6.

Preliminaries Throughout this paper, $\mathbb{Z}$ stands for the set of integers. A positive integer $a>1$ is a prime number if the only positive divisors of it are 1 and $a$. For two integers $a$ and $b$, let $(a, b)$ denote the greatest common divisor of $a$ and $b$. The integers $a$ and $b$ are called co-prime if $(a, b)=1$, and they are called congruent modulo $n$, denoted $a \equiv b \bmod n$, if $n$ divides $a-b$ ( $n$ is an integer too). The notation $a=b$ mod $n$ means that $a$ is the remainder of the integer division of $b$ by $n$. The set of all congruence classes modulo $n$ is denoted $\mathbb{Z}_{n}$. 
Given a random variable $X$ with $n$ outcomes $\left\{x_{1}, \ldots, x_{n}\right\}$, the (Shannon) entropy of $X$, denoted $H(X)$, is defined by

$$
H(X)=\sum_{i=1}^{n} P\left(X=x_{i}\right) \log \frac{1}{P\left(X=x_{i}\right)}
$$

with the mathematical convention $0 \log 0=0$ (here, $P\left(X=x_{i}\right)$ is the probability mass function of the outcome $x_{i}$, and the base of the logarithm is 2). Given two random variables $X$ and $Y, H(X \mid Y)$ stands for the entropy of $X$ conditioned by $Y$.

\section{The Asmuth-Bloom secret sharing scheme and variations}

Given a finite non-empty set $I$ of positive integers and the integers $b_{i}$ and $m_{i}$ for all $i \in I$, the Chinese Remainder Theorem (CRT, for short) [8] states that the system of congruences

$$
x \equiv b_{i} \bmod m_{i}, \quad i \in I
$$

has a unique solution modulo $\prod_{i \in I} m_{i}$, if $m_{i}$ and $m_{j}$ are co-prime for any $i, j \in I$ with $i \neq j$.

One of the applications of CRT is the design of threshold schemes $[2,1,3]$. In this paper we will focus on the threshold scheme in [1] and some of its variants. As all of them are based on sequences of positive integers with special properties, we begin with a few notations and definitions regarding them.

A sequence of co-primes is a sequence

$$
m_{0}, m_{1}, \ldots, m_{n}
$$

of pair-wise co-prime strictly positive integers, where $n \geq 1$. The length of this sequence is $n+1$.

Given two integers $t$ and $n$ with $0<t+1 \leq n$, an Asmuth-Bloom $(t+1, n)$-threshold sequence of co-primes is a sequence of co-primes $m_{0}, m_{1}, \ldots, m_{n}$ which satisfies:

$-m_{0}<m_{1}<\cdots<m_{n}$

- $\prod_{i=1}^{t+1} m_{i}>m_{0} \prod_{i=0}^{t-1} m_{n-i}$ (this is called the Asmuth-Bloom constraint).

Let $t$ and $n$ be integers with $0<t+1 \leq n$. The Asmuth-Bloom $(t+1, n)$-threshold scheme [1] is defined as follows:

(1) parameter setup: consider $m_{0}, m_{1}, \ldots, m_{n}$ an Asmuth-Bloom $(t+1, n)$-threshold sequence of co-primes. The integers $t, n, m_{0}, m_{1}, \ldots, m_{n}$ are public parameters;

(2) secret and share spaces: define the secret space as $\mathbb{Z}_{m_{0}}$ and the share space of the $i$ th participant as $\mathbb{Z}_{m_{i}}$, for all $1 \leq i \leq n$;

(3) secret sharing: given a secret $s$ in the share space, randomly generate $r$ such that $s^{\prime}=s+r m_{0}<\prod_{i=1}^{t+1} m_{i}$. Then, distribute $s$ to the participants by computing $s_{i}=s^{\prime} \bmod m_{i}$ for all $1 \leq i \leq n\left(s_{i}\right.$ is the share of the $i$ th participant, known only by him); 
(4) secret reconstruction: any $t+1$ distinct shares $s_{i_{1}}, \ldots, s_{i_{t+1}}$ can uniquely reconstruct the secret $s$ by computing first the unique solution modulo $\prod_{j=1}^{t+1} m_{i_{j}}$ of the system

$$
x \equiv s_{i_{j}} \bmod m_{i_{j}}, \quad 1 \leq j \leq t+1
$$

and then reducing it modulo $m_{0}$.

The ratio $\left|\mathbb{Z}_{m_{i}}\right| /\left|\mathbb{Z}_{m_{0}}\right|$ is referred to as the information rate of the $i$ th participant, for any $1 \leq i \leq n$.

For the sake of simplicity, we will use the terminology "Asmuth-Bloom sequence of co-primes" ("Asmuth-Bloom threshold scheme") instead of "Asmuth-Bloom $(t+$ $1, n)$-threshold sequence of co-primes" ("Asmuth-Bloom $(t+1, n)$-threshold scheme") whenever it is not important to mention the integers $t$ and $n$.

In order to obtain better security properties of the Asmuth-Bloom threshold scheme, several variants of it were proposed (details about their security will be given at the end of Section 3.1):

1. the variant in [7] considers extended Asmuth-Bloom $(t+1, n)$-threshold sequences of co-primes instead of Asmuth-Bloom $(t+1, n)$-threshold sequences of co-primes, which are simply defined by replacing the Asmuth-Bloom constraint by the following one:

$$
\prod_{i=1}^{t+1} m_{i}>m_{0}^{2} \prod_{i=0}^{t-1} m_{n-i}
$$

2. one of the variants in [4] considers almost $\Theta$-compact sequences of co-primes instead of Asmuth-Bloom sequences of co-primes, where $\Theta \in(0,1)$. These are sequences of co-primes with the property $m_{i} \in\left(x, x+x^{\theta}\right)$ for all $i$, where $x$ linearly depends on $m_{0}$ and $\theta \in(0, \Theta]$;

3. another variation proposed in [4] considers the secret space as being $\mathbb{Z}_{m_{n}}$, while $\mathbb{Z}_{m_{0}}, \ldots, \mathbb{Z}_{m_{n-1}}$ are the share spaces. Moreover, the Asmuth-Bloom sequences of coprimes are replaced by $(n-1, \Theta)$-compact sequences of co-primes, where $\Theta \in(0,1)$. These are sequences of co-primes $m_{0}, m_{1}, \ldots, m_{n}$ which satisfy $m_{0}<m_{1}<\cdots<$ $m_{n}, m_{n} \geq m_{n-1}+2$, and $m_{n}<m_{0}+m_{0}^{\theta}$, for some $\theta \in(0, \Theta]$.

The Asmuth-Bloom threshold scheme and the first two variations above are based on sequences $m_{0}<m_{1}<\cdots<m_{n}$ of co-primes where the integer $m_{0}$ defines the secret space. In the third variation above the secret space is defined by $m_{n}$. In all these cases, all the participants have associated either larger share spaces than the secret space, or smaller share spaces than the secret space. In this context one may think that it would be better to choose $m_{0}$ in the "middle" of the sequence $m_{1}<\cdots<m_{n}$. This would allow for a balanced distribution of the share spaces around the secret space, resulting in a balanced distribution of the participants information rates around 1.

According to this discussion, we will consider a new variation of the AsmuthBloom threshold scheme which is based on $k$-compact sequences of co-primes defined as below. 


\section{Definition 1 ([5]).}

1. A sequence $m_{0}, m_{1}, \ldots, m_{n}$ of pair-wise co-primes is called $(k, \theta)$-compact, where $k \geq 1$ and $\theta \in(0,1)$ are real numbers, if $m_{1}<\cdots<m_{n}$ and $k m_{0}-m_{0}^{\theta}<m_{i}<$ $k m_{0}+m_{0}^{\theta}$ for all $1 \leq i \leq n$.

2. A sequence $m_{0}, m_{1}, \ldots, m_{n}$ of pair-wise co-primes is called $k$-compact if it is $(k, \theta)$ compact for some $\theta \in(0,1)$.

Remark that in a $k$-compact sequence $m_{0}, m_{1}, \ldots, m_{n}$ of co-primes the integer $m_{0}$ may be smaller than $m_{1}$, larger than $m_{n}$, or in between $m_{1}$ and $m_{n}$.

Now, we define a new variation of the Asmuth-Bloom threshold scheme by changing the parameter setup phase into the following one:

(1') parameter setup: consider $m_{0}, m_{1}, \ldots, m_{n}$ a $k$-compact sequence of co-primes. The integers $t, n, m_{0}, m_{1}, \ldots, m_{n}$ are public parameters;

As we will see in Section 4, this new variant of the Asmuth-Bloom threshold scheme meets the best security properties among the variants of the Asmuth-Bloom threshold scheme known so far.

Remark 1. A few words about the relationships between the sequences of co-primes considered above are in order:

1. $(n-1, \Theta)$-compact sequences of co-primes are particular cases of almost $\Theta$-compact sequences of co-primes which, in turn, are particular cases of $k$-compact sequences of co-primes.

2. In [4], compact sequences of co-primes were introduced as being sequences $m_{0}<$ $m_{1}<\cdots<m_{n}$ of co-primes satisfying $m_{i}<m_{0}+m_{0}^{\theta}$, for all $1 \leq i \leq n$ and some $\theta \in(0,1)$. It is clear that compact sequences of co-primes are particular cases of 1-compact sequences of co-primes.

3. Extended Asmuth-Bloom sequences of co-primes are Asmuth-Bloom sequences of co-primes.

4. For sufficiently large $m_{0}, k$-compact sequences $m_{0}, m_{1}, \cdots, m_{n}$ of co-primes with $k>1$ also satisfy the Asmuth-Bloom constraint. Indeed, the Asmuth-Bloom constraint is implied by the inequality

$$
\left(k m_{0}-m_{0}^{\theta}\right)^{t+1}>m_{0}\left(k m_{0}+m_{0}^{\theta}\right)^{t}
$$

As

$$
\lim _{m_{0} \rightarrow \infty} m_{0} \frac{\left(k m_{0}+m_{0}^{\theta}\right)^{t}}{\left(k m_{0}-m_{0}^{\theta}\right)^{t+1}}=\frac{1}{k}
$$

we conclude that the above inequality holds true for sufficiently large $m_{0}$.

We close this section by the following important convention: we use the terminology Asmuth-Bloom threshold scheme based on extended Asmuth-Bloom (almost $\Theta$-compact, $(n-1, \Theta)$-compact, $k$-compact, resp.) sequences of co-primes for the Asmuth-Bloom threshold scheme where the Asmuth-Bloom sequences of co-primes are replaced by extended Asmuth-Bloom (almost $\Theta$-compact, $(n-1, \Theta)$-compact, $k$-compact, resp.) sequences of co-primes. 


\section{Security Issues}

In this section we discuss the security properties an Asmuth-Bloom threshold scheme variant would be desirable to satisfy.

\subsection{Security Properties}

Given the Asmuth-Bloom $(t+1, n)$-threshold scheme and a non-empty set $I \subseteq$ $\{1,2, \ldots, n\}$, consider the random variables $X$ and $Y_{I}$ that take values into the secret space $\mathbb{Z}_{m_{0}}$ and into the share space $\prod_{i \in I} \mathbb{Z}_{m_{i}}$, respectively.

The loss of entropy [6] with respect to $y_{I} \in \prod_{i \in I} \mathbb{Z}_{m_{i}}$, denoted $\Delta\left(y_{I}\right)$, is defined by

$$
\Delta\left(y_{I}\right)=H(X)-H\left(X \mid Y_{I}=y_{I}\right) .
$$

Under the uniform distribution on $\mathbb{Z}_{m_{0}}$, it follows $P(X=s)=1 / m_{0}$ for all $s \in \mathbb{Z}_{m_{0}}$ and, therefore,

$$
H(X)=\sum_{s \in \mathbb{Z}_{m_{0}}} P(X=s) \log \frac{1}{P(X=s)}=\log m_{0}
$$

Definition 2. [6] The Asmuth-Bloom $(t+1, n)$-threshold scheme is called asymptotically perfect if, for any non-empty subset $I \subseteq\{1, \ldots, n\}$ with $|I| \leq t$ and any $\epsilon \in(0,1)$, there exists $m \geq 0$ such that for any Asmuth-Bloom $(t+1, n)$-threshold sequence of co-primes $m_{0}, m_{1}, \ldots, m_{n}$ with $m_{0} \geq m$, the following properties hold:

$-H(X) \neq 0$;

$-\left|\Delta\left(y_{I}\right)\right|<\epsilon$ for any $y_{I} \in \prod_{i \in I} \mathbb{Z}_{m_{i}}$.

The following definition is a slight variation of the asymptotic idealness concept in $[6]$.

Definition 3. Let $0<t+1 \leq n$ be positive integers and $r>0$ be a real number.

1. We say that the information rate of the Asmuth-Bloom $(t+1, n)$-threshold scheme goes asymptotically to $r$ if for any $\epsilon \in(0,1)$ there exists $m \geq 0$ such that for any Asmuth-Bloom $(t+1, n)$-threshold sequence of co-primes $m_{0}, m_{1}, \ldots, m_{n}$ with $m_{0} \geq m$ and any $1 \leq i \leq n$ the following holds:

$$
\left|\frac{\left|\mathbb{Z}_{m_{i}}\right|}{\left|\mathbb{Z}_{m_{0}}\right|}-r\right|<\epsilon .
$$

2. We say that the Asmuth-Bloom $(t+1, n)$-threshold scheme is asymptotically ideal if it is asymptotically perfect and its information rate goes asymptotically to 1. 
Remark 2. Asymptotic perfectness and idealness can naturally be reformulated for the Asmuth-Bloom threshold scheme based on extended Asmuth-Bloom (almost $\Theta$ compact, $(n-1, \Theta)$-compact, $k$-compact, respectively) sequences of co-primes. What we have to do is to replace "Asmuth-Bloom sequence of co-primes" by "extended Asmuth-Bloom sequence of co-primes" ("almost $\Theta$-compact sequence of co-primes", " $(n-1, \Theta)$-compact sequence of co-primes", " $k$-compact sequence of co-primes", respectively).

Having introduced the concepts of asymptotic perfectness and idealness, we recall now some of the results previously obtained:

- the entropy loss in the Asmuth-Bloom threshold scheme is asymptotically upper bounded by 1 [4];

- the Asmuth-Bloom threshold scheme based on almost $\Theta$-compact sequences of co-primes is asymptotically perfect and its information rate goes asymptotically to $2[4]$;

- the Asmuth-Bloom $(t+1, n)$-threshold scheme based on $(n-1, \Theta)$-compact sequences of co-primes is asymptotically ideal [4].

In [7], Kaya and Selcuk have conjectured that replacing the Asmuth-Bloom sequences of co-primes by extended Asmuth-Bloom sequences of co-primes may increase the security of the Asmuth-Bloom threshold scheme. This will be proved in Section 5 .

With respect to $k$-compact sequences of co-primes, we will prove our main result in Section 4 that these kind of sequences lead to a necessary and sufficient condition for asymptotic idealness of the Asmuth-Bloom threshold scheme.

\subsection{Bounding the loss of entropy}

This section is dedicated to finding good approximations for the loss of entropy in the Asmuth-Bloom threshold scheme. We begin by a technical notation intensively used in the rest of the paper.

Given a sequence $m_{0}, m_{1}, \ldots, m_{n}$ of co-primes and a non-empty subset $I \subseteq\{1, \ldots, n\}$, denote by $C(I)$ the integer

$$
C(I)=\left\lfloor\frac{\prod_{i=1}^{t+1} m_{i}}{\prod_{i \in I} m_{i}}\right\rfloor .
$$

The following result is a straightforward adaptation of Lemma 1 in [6] for the case of the Asmuth-Bloom threshold scheme (the proof is omitted).

Lemma 1. [6] The loss of entropy of the Asmuth-Bloom $(t+1, n)$-threshold scheme under a uniform distribution on the secret space satisfies the relations

$$
-\Delta\left(y_{I}\right) \leq \log \frac{m_{0}\left(\left\lfloor\frac{C(I)+1}{m_{0}}\right\rfloor+1\right)}{C(I)} \text {, if } C(I) \neq 0 \text {, }
$$


$-\Delta\left(y_{I}\right)=\log m_{0}$, if $C(I)=0$,

for any non-empty subset $I \subseteq\{1, \ldots, n\}$, any Asmuth-Bloom $(t+1, n)$-threshold sequence of co-primes $m_{0}, m_{1}, \ldots, m_{n}$, and any $y_{I} \in \prod_{i \in I} \mathbb{Z}_{m_{i}}$.

Lemma 1 is an important tool in studying the loss of entropy in the AsmuthBloom threshold scheme. The following result sharpens it by providing a more precise approximation of the loss of entropy in the Asmuth-Bloom threshold scheme.

Lemma 2. The loss of entropy of the Asmuth-Bloom $(t+1, n)$-threshold scheme under a uniform distribution on the secret space satisfies the relation

$$
\Delta\left(y_{I}\right)=\left\{\begin{array}{l}
\log m_{0}+\delta_{1} \frac{\left\lfloor\frac{C_{I}}{m_{0}}\right\rfloor}{C_{I}} \log \frac{\left\lfloor\frac{C_{I}}{m_{0}}\right\rfloor}{C_{I}}+\delta_{2} \frac{\left\lfloor\frac{C_{I}}{m_{0}}\right\rfloor+1}{C_{I}} \log \frac{\left\lfloor\frac{C_{I}}{m_{0}}\right\rfloor+1}{C_{I}}, \quad \text { if } C_{I} \neq 0 \\
\log m_{0}, \text { if } C_{I}=0,
\end{array}\right.
$$

for any non-empty subset $I \subseteq\{1, \ldots, n\}$, any Asmuth-Bloom $(t+1, n)$-threshold sequence of co-primes $m_{0}, m_{1}, \ldots, m_{n}$, any $y_{I} \in \prod_{i \in I} \mathbb{Z}_{m_{i}}$, and some $C_{I} \in\{C(I), C(I)+$ $1\}$, where $\delta_{2}=C_{I} \bmod m_{0}$ and $\delta_{1}=m_{0}-\delta_{2}$.

Proof. Let $I \subseteq\{1, \ldots, n\}$ be a non-empty set and $m_{0}, m_{1}, \ldots, m_{n}$ be an AsmuthBloom $(t+1, n)$-threshold sequence of co-primes.

If $|I|>t$, then $C(I) \in\{0,1\}$ and $H\left(X \mid Y_{I}=y_{I}\right)=0$, which leads to $\Delta\left(y_{I}\right)=$ $H(X)=\log m_{0}$. The choice $C_{I}=C(I)$ concludes the proof in this case.

Let us assume now that $|I| \leq t$, and let $x_{0}$ denote the unique solution modulo $\prod_{i \in I} m_{i}$ obtained by using the CRT over the shares of the participants in $I$. Consider the set

$$
B=\left\{x \in \mathbb{Z}_{\prod_{i=1}^{t+1} m_{i}} \mid x=x_{0}+r \cdot \prod_{i \in I} m_{i}, r \in \mathbb{Z}\right\}
$$

and show that $|B|=C(I)$ or $|B|=C(I)+1$. Indeed, if $x_{0}+r \cdot \prod_{i \in I} m_{i}$ is an element of $B$, then

$$
0 \leq r \leq\left\lfloor\frac{\prod_{i=1}^{t+1} m_{i}-x_{0}}{\prod_{i \in I} m_{i}}\right\rfloor .
$$

As $0 \leq x_{0}<\prod_{i \in I} m_{i}$, we obtain

$$
C(I)-1 \leq\left\lfloor\frac{\prod_{i=1}^{t+1} m_{i}-x_{0}}{\prod_{i \in I} m_{i}}\right\rfloor \leq C(I) .
$$

Therefore, $0 \leq r \leq C(I)-1$ or $0 \leq r \leq C(I)$, depending on $x_{0}$. This shows that $|B|=C(I)$ or $|B|=C(I)+1$. 
Let $C_{I}=|B|$. Then, $B=\left\{x_{0}+r \cdot \prod_{i \in I} m_{i} \mid 0 \leq r<C_{I}\right\}$. According to the division theorem, there exist unique $q \geq 0$ and $0 \leq \delta_{2}<m_{0}$ such that $C_{I}=m_{0} \cdot q+\delta_{2}$. We partition the set $B$ into $B=B_{1} \cup \cdots \cup B_{q} \cup C$, where

$$
B_{i}=\left\{x_{0}+(i-1) m_{0} \cdot \prod_{i \in I} m_{i}, \ldots, x_{0}+\left(i m_{0}-1\right) \cdot \prod_{i \in I} m_{i}\right\}
$$

for all $1 \leq i \leq q$, and

$$
C=\left\{x_{0}+q m_{0} \cdot \prod_{i \in I} m_{i}, \ldots, x_{0}+\left(q m_{0}+\delta_{2}-1\right) \cdot \prod_{i \in I} m_{i}\right\} .
$$

Any two elements in $B_{i}, 1 \leq i \leq q$, are non-congruent modulo $m_{0}$. Indeed, if we consider

$$
x_{0}+\left((i-1) m_{0}+j_{1}\right) \cdot \prod_{i \in I} m_{i} \equiv x_{0}+\left((i-1) m_{0}+j_{2}\right) \cdot \prod_{i \in I} m_{i} \bmod m_{0}
$$

with $j_{1}, j_{2} \in \mathbb{Z}_{m_{0}}$, then $j_{1}=j_{2}$. Similarly, any two elements in $C$ are non-congruent modulo $m_{0}$. As a conclusion, $\left\{x \bmod m_{0} \mid x \in B_{i}\right\}=\mathbb{Z}_{m_{0}}$ for all $1 \leq i \leq q,\{x \bmod$ $\left.m_{0} \mid x \in C\right\} \subset \mathbb{Z}_{m_{0}}$ and $\left|\left\{x \bmod m_{0} \mid x \in C\right\}\right|=\delta_{2}$.

Denoting $\delta_{1}=m_{0}-\delta_{2}$, our conclusion so far is that there exist $\delta_{1}$ elements in $\mathbb{Z}_{m_{0}}$ with the property that each of them is a residue modulo $m_{0}$ of exactly $q$ elements in $B$, and the other $\delta_{2}$ elements in $\mathbb{Z}_{m_{0}}$ have the property that each of them is a residue modulo $m_{0}$ of exactly $q+1$ elements in $B$. According to this,

$$
P\left(X=s \mid Y_{I}=y_{I}\right)=\frac{q}{C_{I}}
$$

for exactly $\delta_{1}$ values $s \in \mathbb{Z}_{m_{0}}$, and

$$
P\left(X=s \mid Y_{I}=y_{I}\right)=\frac{q+1}{C_{I}}
$$

for exactly $\delta_{2}$ values $s \in \mathbb{Z}_{m_{0}}$. Therefore,

$$
\begin{aligned}
\Delta\left(y_{I}\right) & =H(X)-H\left(X \mid Y_{I}=y_{I}\right) \\
& =\log m_{0}-\sum_{s \in \mathbb{Z}_{m_{0}}} P\left(X=s \mid Y_{I}=y_{I}\right) \log \frac{1}{P\left(X=s \mid Y_{I}=y_{I}\right)} \\
& =\log m_{0}+\sum_{s \in \mathbb{Z}_{m_{0}}} P\left(X=s \mid Y_{I}=y_{I}\right) \log P\left(X=s \mid Y_{I}=y_{I}\right) \\
& =\log m_{0}+\delta_{1} \frac{q}{C_{I}} \log \frac{q}{C_{I}}+\delta_{2} \frac{q+1}{C_{I}} \log \frac{q+1}{C_{I}},
\end{aligned}
$$

and the lemma follows from the remark that $q=\left\lfloor\frac{C_{I}}{m_{0}}\right\rfloor$. 
Remark 3. Lemma 1 is a direct consequence of Lemma 2:

$$
\begin{aligned}
\Delta\left(y_{I}\right) & =\log m_{0}+\delta_{1} \frac{\left\lfloor\frac{C_{I}}{m_{0}}\right\rfloor}{C_{I}} \log \frac{\left\lfloor\frac{C_{I}}{m_{0}}\right\rfloor}{C_{I}}+\delta_{2} \frac{\left\lfloor\frac{C_{I}}{m_{0}}\right\rfloor+1}{C_{I}} \log \frac{\left\lfloor\frac{C_{I}}{m_{0}}\right\rfloor+1}{C_{I}} \\
& \leq \log m_{0}+\left(\delta_{1} \frac{\left\lfloor\frac{C_{I}}{m_{0}}\right\rfloor}{C_{I}}+\delta_{2} \frac{\left\lfloor\frac{C_{I}}{m_{0}}\right\rfloor+1}{C_{I}}\right) \log \frac{\left\lfloor\frac{C(I)+1}{m_{0}}\right\rfloor+1}{C(I)} \\
& =\log m_{0}+\frac{\left(\delta_{1}+\delta_{2}\right)\left\lfloor\frac{C_{I}}{m_{0}}\right\rfloor+\delta_{2}}{C_{I}} \log \frac{\left\lfloor\frac{C(I)+1}{m_{0}}\right\rfloor+1}{C(I)} \\
& =\log m_{0}+\frac{m_{0}\left\lfloor\frac{C_{I}}{m_{0}}\right\rfloor+\delta_{2}}{C_{I}} \log \frac{\left\lfloor\frac{C(I)+1}{m_{0}}\right\rfloor+1}{C(I)} \\
& =\log m_{0}+\log \frac{\left\lfloor\frac{C(I)+1}{m_{0}}\right\rfloor+1}{C(I)} \\
& =\log \frac{m_{0}\left(\left\lfloor\frac{C(I)+1}{m_{0}}\right\rfloor+1\right)}{C(I)}
\end{aligned}
$$

if $C(I) \neq 0$ (the inequalities $C(I) \leq C_{I} \leq C(I)+1$ were used).

Remark 4. One can easily see that the result in Lemma 2 (and Lemma 1 as well) does not depend on the type of sequence of co-primes under which the AsmuthBloom threshold scheme is based on. That is, both Lemma 2 and Lemma 1 hold as well if the Asmuth-Bloom threshold scheme is based on extended Asmuth-Bloom (almost $\Theta$-compact, $(n-1, \Theta)$-compact, $k$-compact) sequences of co-primes.

\section{The main result}

In this section we present our main result. Recall first that the concepts of asymptotic perfectness and idealness in the case of the Asmuth-Bloom threshold scheme based on $k$-compact sequences of co-primes are that in Remark 2.

Theorem 1. Let $k \geq 1$ be an integer. The Asmuth-Bloom threshold scheme, under the uniform distribution on the secret space, is asymptotically perfect and its information rate goes asymptotically to $k$ if and only if it is based on $k$-compact sequences of co-primes.

Proof. We prove first the converse of this theorem. Let $0<t+1 \leq n$ be positive integers. Assume that the Asmuth-Bloom $(t+1, n)$-threshold scheme is asymptotically perfect and its information rate goes asymptotically to $k$. Therefore, for any $\epsilon \in(0,1)$ 
there exists $m \geq 0$ such that for any sequence $m_{0}, m_{1}, \ldots, m_{n}$ of co-primes with $m_{0} \geq m$ and any $1 \leq i \leq n$ the following holds:

$$
k m_{0}-\epsilon m_{0}<m_{i}<k m_{0}+\epsilon m_{0} .
$$

We prove that for any $\epsilon \in(0,1)$ there exists $\theta \in(0,1)$ such that $\epsilon m_{0} \leq m_{0}^{\theta}$, where $m_{0}$ is as above. Indeed, if $\epsilon \in\left(m_{0}^{-1}, 1\right)$, then $\theta=1+\log _{m_{0}} \epsilon$ satisfies the required property. If $\epsilon \in\left(0, m_{0}^{-1}\right)$, then any $\theta \in(0,1)$ satisfies the required property.

Therefore, any sequence $m_{0}, m_{1}, \ldots, m_{n}$ of co-primes which satisfies $k m_{0}-\epsilon m_{0}<$ $m_{i}<k m_{0}+\epsilon m_{0}$ for all $1 \leq i \leq n$ and some $\epsilon \in(0,1)$ will also satisfy

$$
k m_{0}-m_{0}^{\theta} \leq k m_{0}-\epsilon m_{0}<m_{i}<k m_{0}+\epsilon m_{0} \leq k m_{0}+m_{0}^{\theta},
$$

where $\theta \in(0,1)$ is defined as above (it depends on $\epsilon$ and $m_{0}$ ). This says that $m_{0}, m_{1}, \ldots, m_{n}$ is $k$-compact.

We prove now that the Asmuth-Bloom $(t+1, n)$-threshold scheme is asymptotically perfect and its information rate goes asymptotically to $k$ if it is based on $k$-compact sequences of co-primes and the secret is uniformly chosen from the secret space.

Asymptotic perfectness. Let $I \subseteq\{1, \ldots, n\}$ denote a non-empty set with $|I| \leq t$, and let $\theta \in(0,1)$. The following cases are to be considered.

Case 1: $|I|<t$. For a given $(k, \theta)$-compact sequence $m_{0}, m_{1}, \ldots, m_{n}$ of co-primes, the property $x-1<\lfloor x\rfloor \leq x$ and Lemma 1 lead to:

$$
\Delta\left(y_{I}\right) \leq \log \frac{m_{1} m_{2} \cdots m_{t+1}+\left(m_{0}+1\right) \prod_{i \in I} m_{i}}{m_{1} m_{2} \cdots m_{t+1}-\prod_{i \in I} m_{i}} .
$$

As, $k m_{0}-m_{0}^{\theta}<m_{i}<k m_{0}+m_{0}^{\theta}$ for any $1 \leq i \leq n$, and $|I| \leq t-1$, the fraction in the right hand side of the above inequality goes to 1 as $m_{0}$ goes to infinity. This shows that for any $\epsilon>0$ there exists $m$ such that $\Delta\left(y_{I}\right)<\epsilon$ if $m_{0} \geq m$.

Case 2: $|I|=t$. We prove first the following Claim which establishes lower and upper bounds for $C_{I}$.

Claim 1. For any $\theta \in(0,1)$ there exists $m$ such that any $(k, \theta)$-compact sequence $m_{0}, m_{1}, \ldots, m_{n}$ with $m_{0} \geq m$ satisfies

$$
k m_{0}-\left(m_{0}^{\theta}+1\right)<C_{I}<k m_{0}+\left(m_{0}^{\theta}+1\right) .
$$

Proof of Claim 1. Let $\theta \in(0,1)$. For any $(k, \theta)$-compact sequence $m_{0}, m_{1}, \ldots, m_{n}$ the following holds:

$$
C_{I} \leq C(I)+1 \leq \frac{\prod_{i=1}^{t+1} m_{i}}{\prod_{i \in I} m_{i}}+1 \leq \frac{\prod_{i=1}^{t+1} m_{i}}{\prod_{i=1}^{t} m_{i}}+1=m_{t+1}+1<k m_{0}+\left(m_{0}^{\theta}+1\right) .
$$

One can easily see that

$$
\lim _{m_{0} \rightarrow \infty} \frac{\prod_{i=1}^{t+1} m_{i}}{m_{0} \prod_{i \in I} m_{i}}=k
$$


and thus, for any $\epsilon>0$ and sufficiently large $m_{0}$ we have

$$
\frac{\prod_{i=1}^{t+1} m_{i}}{m_{0} \prod_{i \in I} m_{i}}>k-\epsilon
$$

Therefore, for any $\epsilon>0$ and sufficiently large $m_{0}$ the following holds:

$$
C_{I} \geq C(I) \geq \frac{\prod_{i=1}^{t+1} m_{i}}{\prod_{i \in I} m_{i}}-1>k m_{0}-\epsilon m_{0}-1
$$

For $\epsilon<m_{0}^{\theta-1}$ we obtain the inequality in Claim 1 .

Let $m_{0}, m_{1}, \ldots, m_{n}$ be a $(k, \theta)$-compact sequence which satisfies Claim 1 . The following two cases are in order:

Case 2.1: $k m_{0}-\left(m_{0}^{\theta}+1\right)<C_{I}<k m_{0}$. Then, $\left\lfloor\frac{C_{I}}{m_{0}}\right\rfloor=k-1$. As $C_{I}=m_{0} \cdot\left\lfloor\frac{C_{I}}{m_{0}}\right\rfloor+\delta_{2}$, we obtain

$$
m_{0}-\left(m_{0}^{\theta}+1\right)<\delta_{2}<m_{0}
$$

which shows that $\delta_{2} / m_{0}$ goes to 1 and $\delta_{1}$ goes to 0 as $m_{0}$ goes to infinity (recall that $\theta$ is fixed). Then, from Lemma 2 it follows

$$
\begin{aligned}
\Delta\left(y_{I}\right)= & \log m_{0}+\delta_{1} \frac{\left\lfloor\frac{C_{I}}{m_{0}}\right\rfloor}{C_{I}} \log \frac{\left\lfloor\frac{C_{I}}{m_{0}}\right\rfloor}{C_{I}}+\delta_{2} \frac{\left\lfloor\frac{C_{I}}{m_{0}}\right\rfloor+1}{C_{I}} \log \frac{\left\lfloor\frac{C_{I}}{m_{0}}\right\rfloor+1}{C_{I}} \\
= & \log m_{0}+\delta_{1} \frac{k-1}{(k-1) m_{0}+\delta_{2}} \log \frac{k-1}{(k-1) m_{0}+\delta_{2}} \\
& +\delta_{2} \frac{k}{(k-1) m_{0}+\delta_{2}} \log \frac{k}{(k-1) m_{0}+\delta_{2}} \\
= & \log m_{0}+\left(\delta_{1} \frac{k-1}{(k-1) m_{0}+\delta_{2}}+\delta_{2} \frac{k}{(k-1) m_{0}+\delta_{2}}\right) \log \frac{k}{(k-1) m_{0}+\delta_{2}} \\
& +\delta_{1} \frac{k-1}{(k-1) m_{0}+\delta_{2}}\left(\log \frac{k-1}{(k-1) m_{0}+\delta_{2}}-\log \frac{k}{(k-1) m_{0}+\delta_{2}}\right) \\
= & \log m_{0}+\log \frac{k}{(k-1) m_{0}+\delta_{2}}+\delta_{1} \frac{k-1}{(k-1) m_{0}+\delta_{2}} \log \frac{k-1}{k} \\
= & \log \frac{k m_{0}}{(k-1) m_{0}+\delta_{2}}+\delta_{1} \frac{k-1}{(k-1) m_{0}+\delta_{2}} \log \frac{k-1}{k} \\
= & \log \frac{k m_{0}}{k m_{0}-\delta_{1}}+\delta_{1} \frac{k-1}{k m_{0}-\delta_{1}} \log \frac{k-1}{k}
\end{aligned}
$$


if $k>1$, and

$$
\begin{aligned}
\Delta\left(y_{I}\right) & =\log m_{0}+\delta_{1} \frac{\left\lfloor\frac{C_{I}}{m_{0}}\right\rfloor}{C_{I}} \log \frac{\left\lfloor\frac{C_{I}}{m_{0}}\right\rfloor}{C_{I}}+\delta_{2} \frac{\left\lfloor\frac{C_{I}}{m_{0}}\right\rfloor+1}{C_{I}} \log \frac{\left\lfloor\frac{C_{I}}{m_{0}}\right\rfloor+1}{C_{I}} \\
& =\log \frac{m_{0}}{\delta_{2}} \\
& =\log \frac{m_{0}}{m_{0}-\delta_{1}}
\end{aligned}
$$

if $k=1$ (the convention $0 \log 0=0$ was used).

As both $\delta_{1} /\left(k m_{0}-\delta_{1}\right)$ and $\delta_{1} / m_{0}$ goes to 0 as $m_{0}$ goes to infinity, we deduce that $\Delta\left(y_{I}\right)$ goes to 0 as $m_{0}$ goes to infinity.

Case 2.2: $k m_{0} \leq C_{I}<k m_{0}+\left(m_{0}^{\theta}+1\right)$. Then, $\left\lfloor\frac{C_{I}}{m_{0}}\right\rfloor=k$. As $C_{I}=m_{0} \cdot\left\lfloor\frac{C_{I}}{m_{0}}\right\rfloor+\delta_{2}$, we obtain

$$
0 \leq \delta_{2}<m_{0}^{\theta}+1
$$

which shows that $\delta_{2} / m_{0}$ goes to 0 and $\delta_{1}$ goes to 1 as $m_{0}$ goes to infinity (recall that $\theta$ is fixed). Then, from Lemma 2 it follows:

$$
\begin{aligned}
\Delta\left(y_{I}\right)= & \log m_{0}+\delta_{1} \frac{\left\lfloor\frac{C_{I}}{m_{0}}\right\rfloor}{C_{I}} \log \frac{\left\lfloor\frac{C_{I}}{m_{0}}\right\rfloor}{C_{I}}+\delta_{2} \frac{\left\lfloor\frac{C_{I}}{m_{0}}\right\rfloor+1}{C_{I}} \log \frac{\left\lfloor\frac{C_{I}}{m_{0}}\right\rfloor+1}{C_{I}} \\
= & \log m_{0}+\delta_{1} \frac{k}{k m_{0}+\delta_{2}} \log \frac{k}{k m_{0}+\delta_{2}}+\delta_{2} \frac{k+1}{k m_{0}+\delta_{2}} \log \frac{k+1}{k m_{0}+\delta_{2}} \\
= & \log m_{0}+\left(\delta_{1} \frac{k}{k m_{0}+\delta_{2}}+\delta_{2} \frac{k+1}{k m_{0}+\delta_{2}}\right) \log \frac{k}{k m_{0}+\delta_{2}} \\
& +\delta_{2} \frac{k+1}{k m_{0}+\delta_{2}}\left(\log \frac{k+1}{k m_{0}+\delta_{2}}-\log \frac{k}{k m_{0}+\delta_{2}}\right) \\
= & \log m_{0}+\log \frac{k}{k m_{0}+\delta_{2}}+\delta_{2} \frac{k+1}{k m_{0}+\delta_{2}} \log \frac{k+1}{k} \\
= & \log \frac{k m_{0}}{k m_{0}+\delta_{2}}+\delta_{2} \frac{k+1}{k m_{0}+\delta_{2}} \log \frac{k+1}{k}
\end{aligned}
$$

As $\delta_{2} /\left(k m_{0}+\delta_{2}\right)$ goes to 0 as $m_{0}$ goes to infinity, we deduce that $\Delta\left(y_{I}\right)$ goes to 0 as $m_{0}$ goes to infinity.

Information rate. Given $\theta \in(0,1)$ and $m_{0}, m_{1}, \ldots, m_{n}$ a $(k, \theta)$-compact sequence of co-primes, we have

$$
\frac{k m_{0}-m_{0}^{\theta}}{m_{0}}<\frac{\left|\mathbb{Z}_{m_{i}}\right|}{\left|\mathbb{Z}_{m_{0}}\right|}=\frac{m_{i}}{m_{0}}<\frac{k m_{0}+m_{0}^{\theta}}{m_{0}}
$$

which shows that the information rate goes to $k$ as $m_{0}$ goes to infinity. 
Corollary 1. The Asmuth-Bloom threshold scheme is asymptotically ideal with respect to the uniform distribution on the secret space if and only if it is based on 1-compact sequences of co-primes.

Proof. This is the case $k=1$ in Theorem 1 .

Remark 5. Choosing $m_{1}<\cdots<m_{n}$ in a compact interval centered at $m_{0}$, offers the maximum of optimality with respect to the asymptotic idealness of the AsmuthBloom $(t+1, n)$-threshold scheme.

\section{Extended Asmuth-Bloom sequences of co-primes}

In this section we focus on the Asmuth-Bloom threshold scheme based on extended Asmuth-Bloom sequences of co-primes [7].

Theorem 2. The Asmuth-Bloom threshold scheme based on extended Asmuth-Bloom sequences of co-primes is asymptotically perfect under the uniform distribution on the secret space.

Proof. Let $0<t+1 \leq n$ be positive integers and $I \subseteq\{1, \ldots, n\}$ be a non-empty set with $|I| \leq t$. Then, $C(I)>m_{0}^{2}$ for any extended Asmuth-Bloom $(t+1, n)$-threshold sequence of co-primes $m_{0}, m_{1}, \ldots, m_{n}$, and Lemma 1 (see also Remark 4) leads to

$$
\Delta\left(y_{I}\right) \leq \log \frac{C(I)+2 m_{0}}{C(I)}
$$

which shows that the entropy loss goes to zero as $m_{0}$ goes to infinity.

Remark 6. One may define extended Asmuth-Bloom $(t+1, n)$-threshold sequences of co-primes in a more liberal way by requiring

$$
\prod_{i=1}^{t+1} m_{i}>m_{0}^{1+\theta} \prod_{i=0}^{t-1} m_{n-i}
$$

for some real number $\theta>0$.

The result in Theorem 2 holds in this case too. Moreover, $m_{0}^{1+\theta}<m_{1}$ which shows that the information rate of the first participant (and in fact, of all participants) is greater than $m_{0}^{\theta}$.

\section{Conclusions}

In this paper we presented a necessary and sufficient condition for the asymptotic idealness of the Asmuth-Bloom threshold scheme. Namely, it was shown that this scheme is asymptotically ideal if and only if it is based on 1-compact sequences of 
co-primes. Moreover, a comprehensive analysis of the known variants of the AsmuthBloom threshold scheme is provided, clarifying the security properties achieved by each of them.

Although the case $k=1$ in Theorem 1 represents the main result regarding the security of the Asmuth-Bloom threshold scheme, it would be interesting to extend the characterization in this theorem to the case where $k>1$ is a real number but not an integer one.

\section{References}

1. C. A. Asmuth and J. Bloom, "A modular approach to key safeguarding," IEEE Transactions on Information Theory, vol. 29, no. 2, pp. 208-210, Mar. 1983, the paper was presented at the National Telecommunications Conference, Houstan, Dec. 1980.

2. M. Mignotte, "How to share a secret?" in Workshop on Cryptography, ser. Lecture Notes in Computer Science, T. Beth, Ed., vol. 149, Burg Feuerstein, 1982, pp. 371-375.

3. O. Goldreich, D. Ron, and M. Sudan, "Chinese remaindering with errors," IEEE Transactions on Information Theory, vol. 46, no. 4, pp. 1330-1338, Mar. 2000.

4. M. Barzu, F. L. Ţiplea, and C. C. Drăgan, "Compact sequences of co-primes and their applications to the security of CRT-based threshold schemes," Information Sciences, vol. 240, pp. 161-172, 2013.

5. F. L. Ţiplea and C. C. Drăgan, "A necessary and sufficient condition for the asymptotic idealness of the GRS threshold secret sharing scheme," Information Processing Letters, vol. 114, no. 6, pp. 299 - 303, 2014.

6. M. Quisquater, B. Preneel, and J. Vandewalle, "On the security of the threshold scheme based on the Chinese remainder theorem," in Public Key Cryptography, ser. Lecture Notes in Computer Science, D. Naccache and P. Paillier, Eds., vol. 2274. Springer, 2002, pp. 199-210.

7. K. Kaya and A. A. Selçuk, "Threshold cryptography based on Asmuth-Bloom secret sharing," Information sciences, vol. 177, no. 19, pp. 4148-4160, 2007.

8. C. Ding, D. Pei, and A. Salomaa, Chinese Remainder Theorem. Applications in Computing, Coding, Cryptography. World Scientific Publishing, 1996. 